\title{
Synergistic Smart Morphing Aileron
}

\author{
Alexander M. Pankonien ${ }^{1}$ \\ University of Michigan, Ann Arbor, MI, 48109, USA \\ Cassio T. Faria ${ }^{2}$ \\ Virginia Tech, Blacksburg, VA, 24061, USA \\ and \\ Daniel J. Inman ${ }^{3}$ \\ University of Michigan, Ann Arbor, MI, 48109, USA
}

\begin{abstract}
Smart materials have been widely applied in morphing aerospace structures, improving performance and reducing weight and mechanical complexity when compared to conventional actuators. However these novel components also have intrinsic limitations that restrict the capabilities of the resultant structures. This paper introduces the Synergistic Smart Morphing Aileron (SSMA), which overcomes the individual limitations of the constituent materials. Independent control surfaces mechanisms, driven by shape memory alloy and micro-fiber composite actuators are presented, culminating in a fused design of both technologies that improves the overall actuation range and bandwidth. The concept of synergetic use of smart material is not widely diffused in the scientific community and it is added upon by this study.
\end{abstract}

\section{Nomenclature}

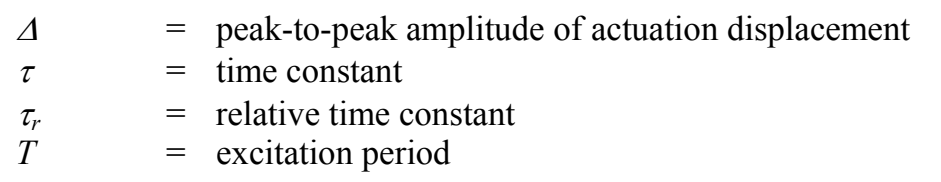

\section{Introduction}

T nature, variable camber allows birds to maximize aerodynamic efficiency over a range of flight conditions by 1 minimizing drag losses ${ }^{1}$. Aircraft designers have sought to imitate the efficiency of avian designs, starting with wing warping by the Wright Brothers and continuing to the modern, discrete aileron. However, these designs are only rough approximations of true control over the airfoil shape. Smoothly morphing trailing edges with integrated actuators are the next step toward continuous airfoil profile control.

It has been shown that smooth, morphing airfoils exhibit an aerodynamic advantage over airfoils with conventional, discrete trailing edge control surfaces ${ }^{2,3,4}$. The smooth surface of a morphing aileron leads to delayed transition and reduced separation leading to lower drag over the airfoil and increased aerodynamic performance ${ }^{5}$. The aerodynamic advantage of morphing trailing edges increases further when losses due to aileron hinge gaps are considered. These losses contribute to the drag of the aircraft limiting the performance and range ${ }^{5}$. For UAVs and long-endurance aircraft, these losses can be substantial with respect to the total power availability, motivating research in morphing concepts.

Initially, morphing aileron designs for changing airfoil camber utilized purely mechanical solutions with conventional actuators, such as flexible fowler flaps ${ }^{2}$ had additional mechanical complexity that made the devices prohibitive in maintenance and weight ${ }^{6}$. Other novel approaches, such as using compliant mechanisms ${ }^{7}$, address this issue by reducing the number of moving components, but still have separate actuators and structures. These

${ }^{1}$ Graduate Research Assistant, Department of Aerospace Engineering, 1320 Beal Avenue, AIAA Student Member.

${ }^{2}$ Graduate Research Assistant, Department of Mechanical Engineering, 100 Randolph Hall, AIAA Student Member.

${ }^{3}$ Collegiate Professor and Chair, Department of Aerospace Engineering, 1320 Beal Avenue, AIAA Fellow. 
actuators are commonly embedded within the airfoil structure with an inactive compliant material as the skin, forming a continuous morphing control surface. Developments in "smart" materials, which function as both actuator and structure, show promise in maximizing the design efficiency by reduce the mechanical complexity.

Previously, morphing aircraft designs using smart materials have focused on single-material actuation concepts. Typically the actuation materials used are shape memory alloys (SMA), shape memory polymers (SMP), piezoelectric ceramics such as Lead zirconate titanate (PZT), or piezoelectric composites which use PZT, such as the Macro Fiber Composite (MFC). Many different applications have been developed that use a single smart material to replace the role of a conventional actuator to change span, chord, sweep, twist, bend and camber of the wing ${ }^{6}$.

By focusing on the implementation of a single smart material, the design complexity is reduced; however, the actuation limitations of the design are governed largely by the limitations of the specific smart material being used. As seen in Figure 1 below, neither SMAs nor PZT exhibits superior actuation stress, strain, bandwidth, and specific work. Although piezoelectric materials have large actuation bandwidth, their actuation strains are typically limited $(0.2 \%$ or less). Opposingly, shape memory alloys have large actuation stress and strains, but very low actuation frequencies $(10 \mathrm{~Hz}$ or less in heating and often much slower in cooling). Consequently, actuation designs using piezoelectric materials typically have small displacements or force but quick response and conversely, designs using shape memory materials have large displacements or force but a very slow response.
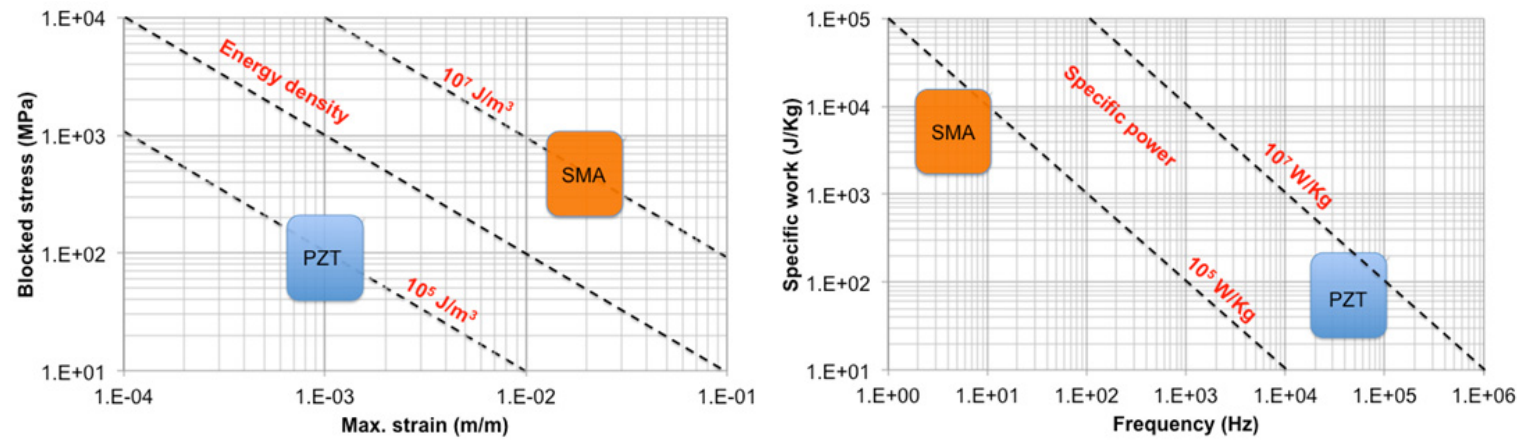

Figure 1. Smart Material Actuator Comparison (Adapted from - NASA/JPL/EAP website)

Ideally, a design would combine several smart materials in a synergistic manner so that the overall actuation capabilities would realize performance beyond that of the individual materials. However, the relative complexity of designing a structure with multiple smart actuators has limited the number of multi-material designs in literature. Still, the combined advantage of using multiple materials has motivated several designs that utilize the mismatch in material properties. For example, shape memory materials can be used as both fiber and matrix in a material, so that all elements of the composite can actuate to a greater extent on the same timescale ${ }^{8}$. Alternatively, a piezoelectric matrix was used to induce stress transformations within shape memory alloy inclusions in a piezo-SMA composite, increasing the "actuation" speed of the slower SMA". Finally, a flapping wing robot used shape memory polymers to control the stiffness of a compliant structure under actuation by a piezoelectric bimorph, effectively altering the compliance of the structure over two different timescales ${ }^{10}$. It is this concept that motivates the current design for improved aileron performance.

\section{Mechanism Description}

The proposed concept uses multiple smart materials in a single structure to provide increased control capabilities beyond that of the individual materials, without prohibitively increasing design complexity and thus weight. Leveraging the differing actuation time scales and ranges of shape memory alloys and piezoelectric fibers, the design uses a combination of antagonistic MFCs and shape memory alloy wire actuators to control a morphing aileron. The SMA wires controls larger angle deflections at the root of the aileron over a slower timescale while the MFCs control precise conformal variations over a quicker timescale. The design is further explained in the following sections by discussing the implementation details of the MFC and SMA separately, and then combined in the final design.

\section{A. MFC-Actuated Flexure Box Design}

Of the many various smart materials developed over the past few decades, piezoelectric ceramics have been widely used due to their high bandwidth, large actuation force, and the relative ease of actuation control through 
applied voltage. However, conventional monolithic ceramics are brittle and rigid with low actuation strains, making implementation in flexible skins and large displacement actuators difficult. Additionally, high voltages must be applied for the piezoelectric material to maintain its deformed state. The development of the Active Fiber Composite (AFC) allowed these piezo-ceramics to be manufactured as thin fibers within an epoxy composite that were relatively flexible and could achieve higher actuation strains than conventional monolithic configurations ${ }^{11}$. Recently, the development of the Macro-Fiber Composite (MFC) changed the production process from extruding cylindrical fibers to slicing wafers, lowering the production cost and improving market accessibility ${ }^{12}$.These MFCs have seen significant use in Micro Air Vehicles as actuators for flight control by either inducing strains in the existing structure or directly serving as the control surface ${ }^{13}$. Despite these developments, MFCs require even higher voltages to actuate (up to $1.5 \mathrm{kV}$ ) that must be constantly applied to maintain the deformed shape, though with very low power consumption.

Previous work with MFCs developed the Cascading Bimorph variable-camber airfoil concept, shown in Figure 2 ${ }^{14}$. This design additional thickness to the airfoil and improved boundary conditions over previous MFC bimorph designs. Elastomer skin spanned the gap between the compliant box and the leading edge of the airfoil maintaining surface continuity. The actuations results showed an improvement of lift curve slope by $72 \%$ over a rigid change in angle of attack and were compared with other common airfoils. The results were not compared conventional control surfaces due to prohibitive nature of the experimental complexity; however, no aeroelastic effects were seen for free-stream flow speeds around $15 \mathrm{~m} / \mathrm{s}$ with a Reynold's number of $1.27 \times 10^{5}$, and for forced fan speeds of $45 \mathrm{~m} / \mathrm{s}$.
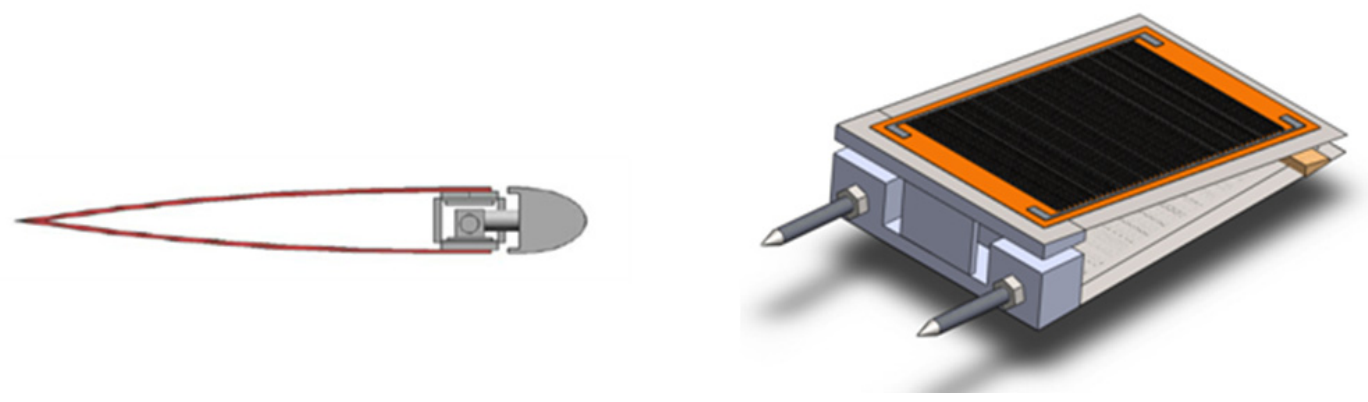

Figure 2. Novel Morphing Compliant Box Airfoil ${ }^{14}$

Figure 3: Flexure Box Aileron ${ }^{15}$

Expanding upon the original concept, the cascading bimorph airfoil design was modified to create the Flexure Box aileron, shown in Figure $3^{15}$. The new design allows for simplified implementation with conventional airfoil profiles by allowing the upper and lower surface of the aileron to be manufactured at independent small angles. Additionally, the conventional hinges from the original design were also replaced with flexure mechanisms. This new design is created by replacing the sides of the compliant box with an elastomeric material while leaving the top and bottom as rigid ABS plastic. The upper and lower surfaces remain as MFCs bonded to a thin metal substrate and a stub is added to the trailing edge to ensure the correct angle. Using continuous, flexible structures eliminates positioning errors from nonlinearities caused by lag and friction within the hinge joints and has the added advantage of making the design amenable for stiffness optimization about expected loading conditions. The resultant design is a high-frequency, high-resolution morphing airfoil section that maintains surface continuity and allows for precise but limited aerodynamic control.

\section{B. SMA-Actuated Hinge}

Shape memory alloys (SMAs) have been, in the past decade, extensively employed as an actuator for a wide range of applications ranging from medical instrumentation ${ }^{16}$, thermal regulators ${ }^{17}$, robotics ${ }^{18}$ and much more. In cases where SMAs are used, the characteristics demanded from the actuator are very similar: high force, large displacement and low actuation frequency. Besides being able to completely satisfy the actuator design requirements, SMAs have the advantage of being in a solid state form, i.e. no moving parts, which increases the overall system reliability by reducing the amount of components subjected to failure.

To activate the shape memory property the material undergoes a solid-to-solid microstructural phase change. This complex transformation is driven by temperature and mechanical stresses. A more detailed description of this material's properties and thermomechanical behavior is given by Brinson ${ }^{19}$. The transformation's dependency on 
heat flow inside the active element has two significant limitations that limit the application of this material: low energy efficiency and slow actuation cycles.

To reduce energy loss and improve time response, primarily thin SMA materials are used to their relative lack of complexity and high surface area to volume ratio, which aids in the cooling process. Additionally, the use of thin wires allows for the heating to be achieved by electrical currents. For example, several actuation concepts include: an elastic substrate embedded with SMA wires ${ }^{20}$, ribbons ${ }^{21}$, thin films ${ }^{4}$; elastic structures with a single SMA wire ${ }^{22}$, elastic hinges driven by a pair of SMA wires ${ }^{23}$; and hinges rotated by a pair of SMA wires ${ }^{24},{ }^{25}$.

As proposed by Faria, et al (2012) ${ }^{25}$, and illustrated by Figure 4, the camber line of a sectioned airfoil can be altered by antagonistically heating and cooling the SMA wires located above and below the connecting hinge. By heating one of the wires in an initially deformed state, a reduction in wire length due to transformation strain in the SMA will create a relative rotation between structures while straining the opposing, cooling wire. After the actuation is complete and the desired angular position is reached, no additional energy is required to maintain the deformed shape. However, due to the relatively slow cooling process in the SMA wires during the phase change, the mechanism has a low frequency response compared to MFCs.
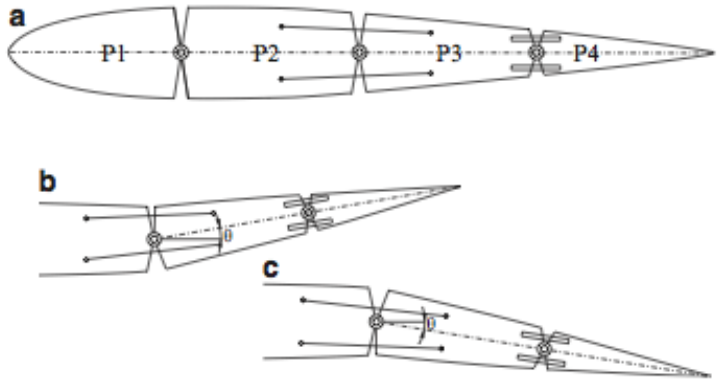

Figure 4. SMA-actuated hinge concept

a) Initial unactuated position (but strained SMA wires); b) Angular displacement on the counter-clockwise by heating of the upper wire; c) actuation in the reverse direction by heating the lower SMA wire.

\section{Combined Design}

Both the MFC and SMA designs presented in the previous sections have been previously employed in literature as independent mechanisms to actively change the camber line of an airfoil. However, as discussed earlier, both actuators operate over different timescales using different mechanisms. By integrating the two technologies a versatile structure for a Synergetic Smart Morphing Aileron (SSMA) is created, as illustrated in Figure 5.

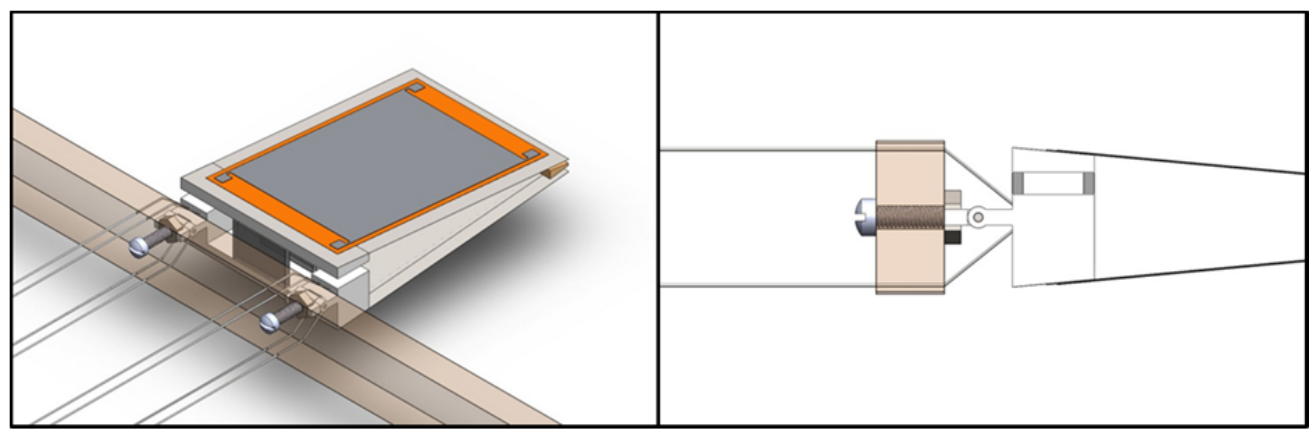

Figure 5. SSMA Concept

As seen in the figure above for the combined SSMA concept, both the flexure box aileron and SMA-actuated hinge are combined into a single concept that uses both without significantly increasing the size or complexity of the device. The wires are used to actuated about a hinge that is located between the compliant box and the mounting spar (pictured here as translucent brown). The SMA wires are anchored in the bottom of the previously-fixed Flexure Box Aileron and run through the mounting surface where appropriate electrical leads and control circuit can be applied (not pictured). Similarly, electrical wires for controlling the MFCs can run through a hole in mounting surface to a control circuit. The material spanning the gap above the hinge can be determined by the needs of the specific application. For example, in a small remote controlled aircraft, the morphing control surface could serve as 
a morphing aileron or rudder mounted to the rear spar of the wing, using an elastomer, such as latex, as the spanning material.

\section{Experimental Setup}

The SSMA concept was constructed for a representative airfoil section to experimentally evaluate the ranges and timescales associated with the constituent smart materials, seen in Figure 6. A NACA 0012 airfoil with a $305 \mathrm{~mm}$ chord was chosen with a mounting spar location at $153 \mathrm{~mm}$ aft of the leading edge. A Keyence LKG-402 laser displacement sensor measured the displacement of the SSMA at $142 \mathrm{~mm}$ aft of the rear spar. Both the Flexure Box mechanism and the hinge for the SMA, described in previous sections, were created using the Objet Connex 500 multi-material 3D printer. Although the Flexure box is completely solid-state, the hinge for the SMA was constructed using a discrete hinge, due to the compressive effect of the SMA wires on the hinge. Based on the volume of smart materials utilized in this application their masses were calculated to be about 0.1 grams for the SMA and 8 grams for the PZT in the MFC, reflecting the comparatively higher specific work associated with SMAs.

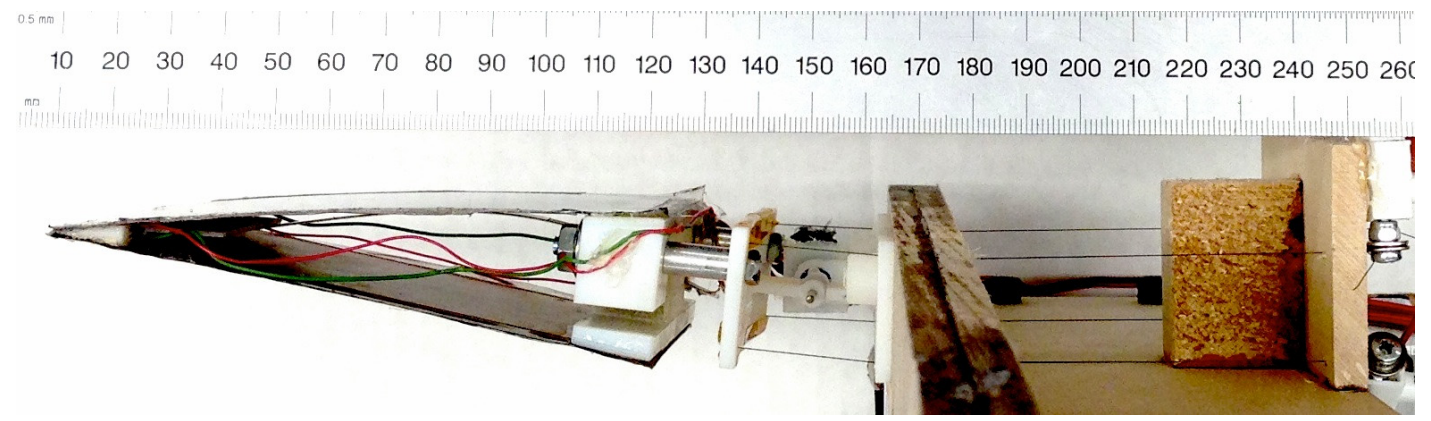

Figure 6: Experimental Construction of SSMA Concept

Two $200 \mu \mathrm{m}$ diameter, SMA wires were used in the antagonistic configurationl about the SMA hinge. The aft mounting location for SMA wires was changed to a flat plate on which the flexure box concept was also mounted. This change allowed the top and bottom SMA wires to exert equal moments about the hinge, independent of the Flexure Box configuration. The SMA wires were anchored at a representative front spar, located at $50 \mathrm{~mm}$ chord. Each $238 \mathrm{~mm}$ wire ran from the front spar, through the rear spar to the aft mounting location on the flat plate, and then back through the rear spar to the front spar, to close the electrical connection. A custom-built switching circuit used a $10 \mathrm{Amp}$ relay to alternate a constant current of 0.6 Amps at 5 Volts between the two wires for heating. No active cooling system was used. Although variable-current power sources are available, the electrical circuit was chosen for its simplicity and ease of implementation.

The Flexure Box Aileron was manufactured with two unimorphs, each consisting of one M8557-P1 MFC from Smart Material Corportation, bonded using 3M Scotch-Weld DP-460 to $0.025 \mathrm{~mm}$ thick stainless steel shim. The two MFCs unimorphs were operated together in beding using an AVID Dual Channel MFC Bimorph High-Voltage Driver. The driver board applied voltage to the unimorphs in a 3:-1 ratio so that the maximum applied voltages were $1500 \mathrm{~V}$ and $-500 \mathrm{~V}$ for the opposing unimorphs.

The design also allowed for integration of embedded sensors for closed loop position control, important for hysteretic actuators undergoing aerodynamic loads. A potentiometer was integrated into the discrete hinge for the SMA to allow for calibrated angular rotation to be measured. The MFC bimorph, having no discrete hinges, required a slightly more elegant solution. A unidirectional Flex sensor, which changes its resistance as it is flexed, was installed in a pre-flexed configuration in the Flexure Box concept, pictured below in Figure 7. Both sensors were implemented in separate Wheatstone bridge configurations, by which the position of the various actuators could be determined after calibration with the laser. These sensors will allow for implementation of a closed loop synergistic controller in future studies; however, they were not used in the current study due to simplicity of implementation and measurement with the laser displacement sensor. 

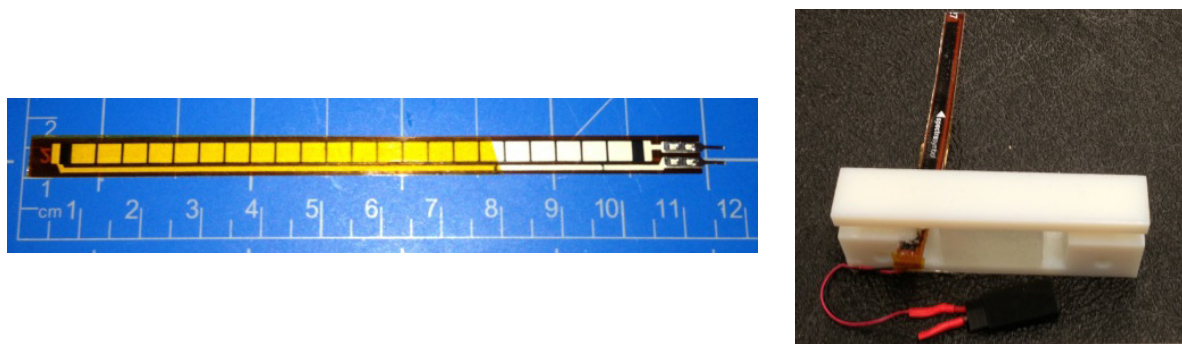

Figure 7. Flex Sensor, implemented in Flexure Box concept.

\section{Data Processing and Results}

The performance of each active mechanism was evaluated using square wave excitation for a range of frequencies, with particular interest in two response aspects: maximum amplitude of motion and the mechanism speed. Experimentally measuring this later aspect of the response for different square-wave frequencies is complicated as the velocity exponentially decays to zero, as illustrated in Fig. 8. Based on the response of first order systems, e.g. an RC electrical circuit, the time constant $(\tau)$ was chosen to be part of metric to evaluate the actuator speed $^{26}$. Figure 8 below compiles information about the variables used to describe the response of the active mechanism and the excitation signal.

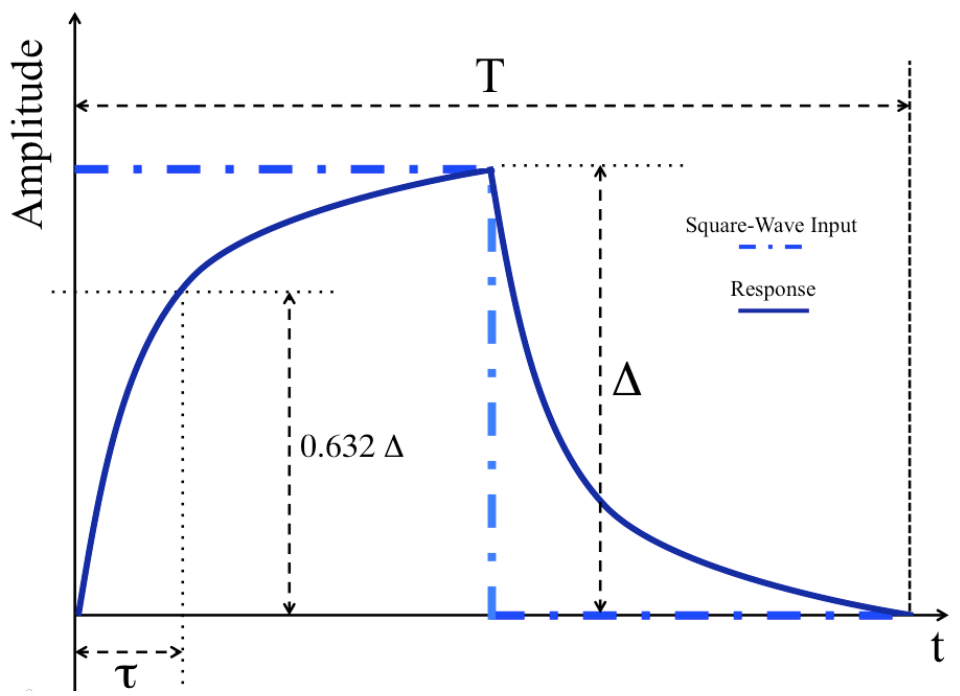

Figure 8. Illustration of the square wave time response

The response peak-to-peak amplitude $(\Delta)$ is an intuitive concept, while the time constant $(\tau)$ is a value derived from the exponential reaching condition for a first order system ${ }^{26}$.To allow a direct comparison between responses for different input frequencies the time constant is normalized by the excitation period $(T)$, a concept mathematically described by Eq. (1).

$$
\tau_{r}=\frac{\tau}{T}
$$

The expression above also implies that the relative time constant $\left(\tau_{r}\right)$ has a upper limit of 0.5 because at half of the wave period the square wave reverses direction. By plotting the response amplitude $(\Delta)$ and relative time constant (RTC) against the excitation frequency, a Square Wave Response Function (SWRF) is obtained. This plot compiles the information of amplitude and velocity behavior for the actuator mechanisms allowing a direct performance comparison between them. The SWRF was generated by measuring the steady-state response of the 
system over a relevant range of finite frequencies. The following plots show the SWRF function with error bars for the standard deviation of the measured values within each test.

The first active mechanism to be tested only actuated the shape memory alloy wires. The system was excited with square waves from $0.005 \mathrm{~Hz}$ to $1 \mathrm{~Hz}$. The resulting SWRF is presented below by Figure 9 .
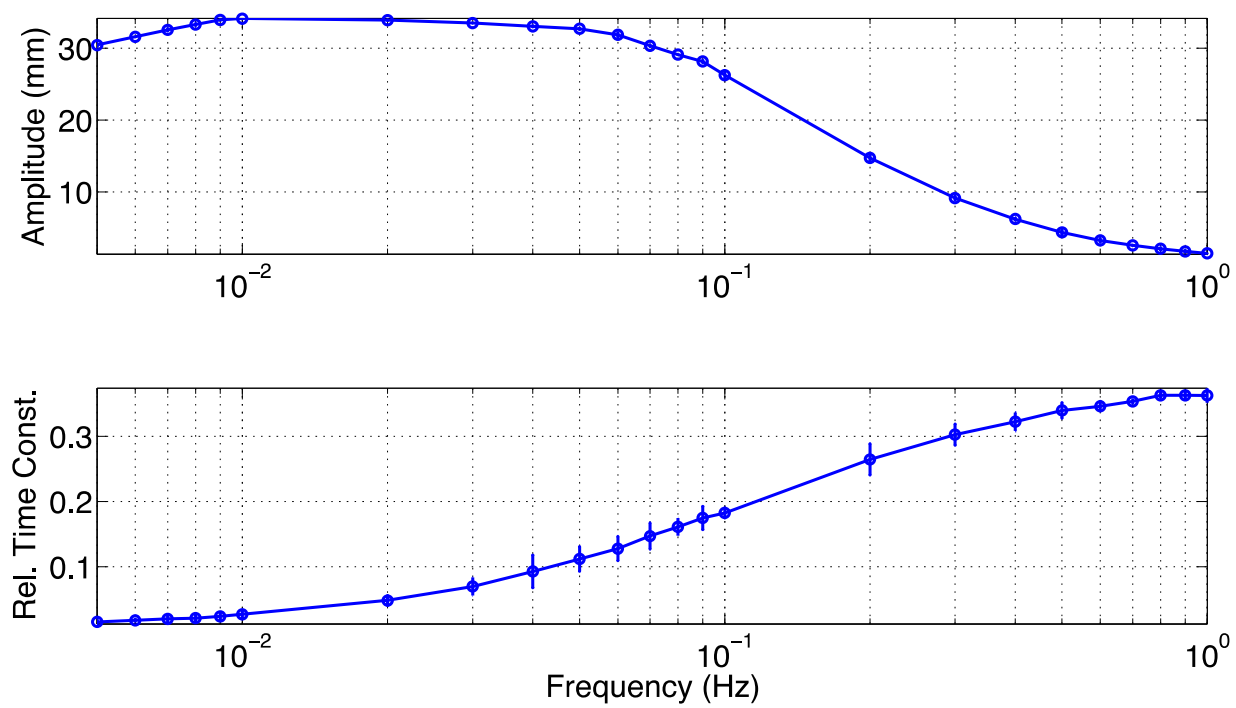

Figure 9. Experimental SWRF plots for the SMA active mechanism

For the lower frequencies the SMA-based mechanism has a near zero relative time constant which means that the device reaches the vicinity of the maximum amplitude in a small time when compared to the input period, shown in Figure 10(a). As the frequency increases, the actuation amplitude reduces and RTC increases indicating that the mechanism performance is decreasing. At higher frequencies, the slew rate becomes insufficient to allow the actuator to reach the commanded bounds in the time period and the mechanism rate-saturates, characterized by a low peak-to-peak amplitude and high RTC, Figure 10(b).
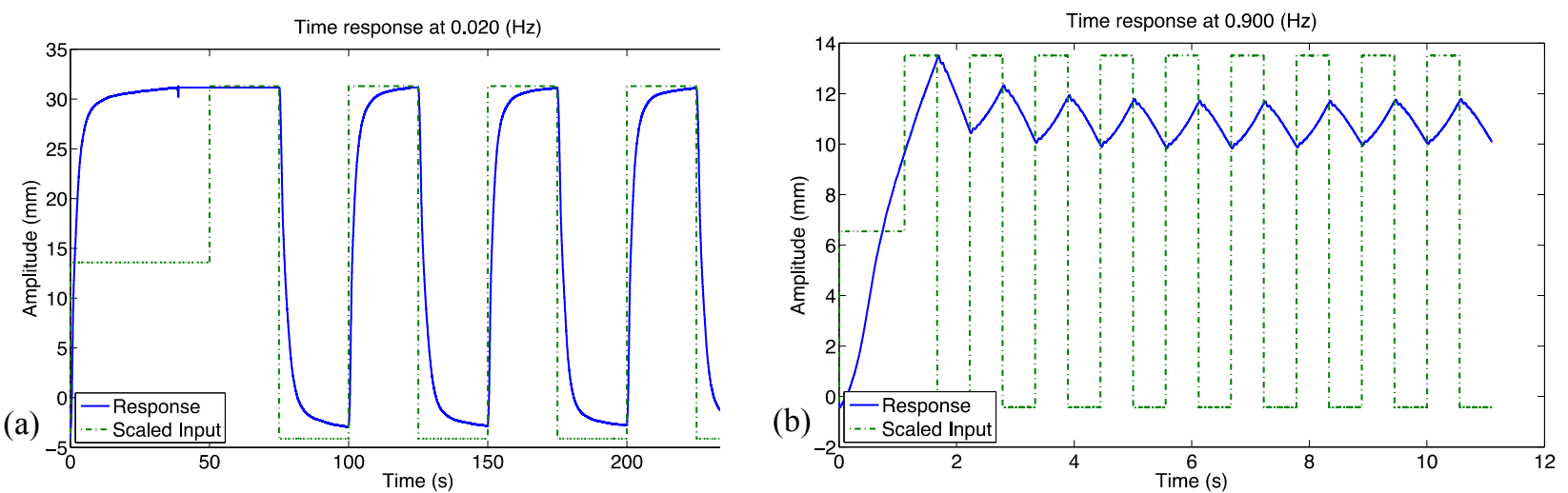

Figure 10. SMA-based actuator time response for (a) $0.02 \mathrm{~Hz}$ (b) $0.9 \mathrm{~Hz}$.

For the MFC-based mechanism, the tests were performed for a different range square wave frequencies: from $0.1 \mathrm{~Hz}$ to $10 \mathrm{~Hz}$. The resulting SWRF is presented in Figure 11. 

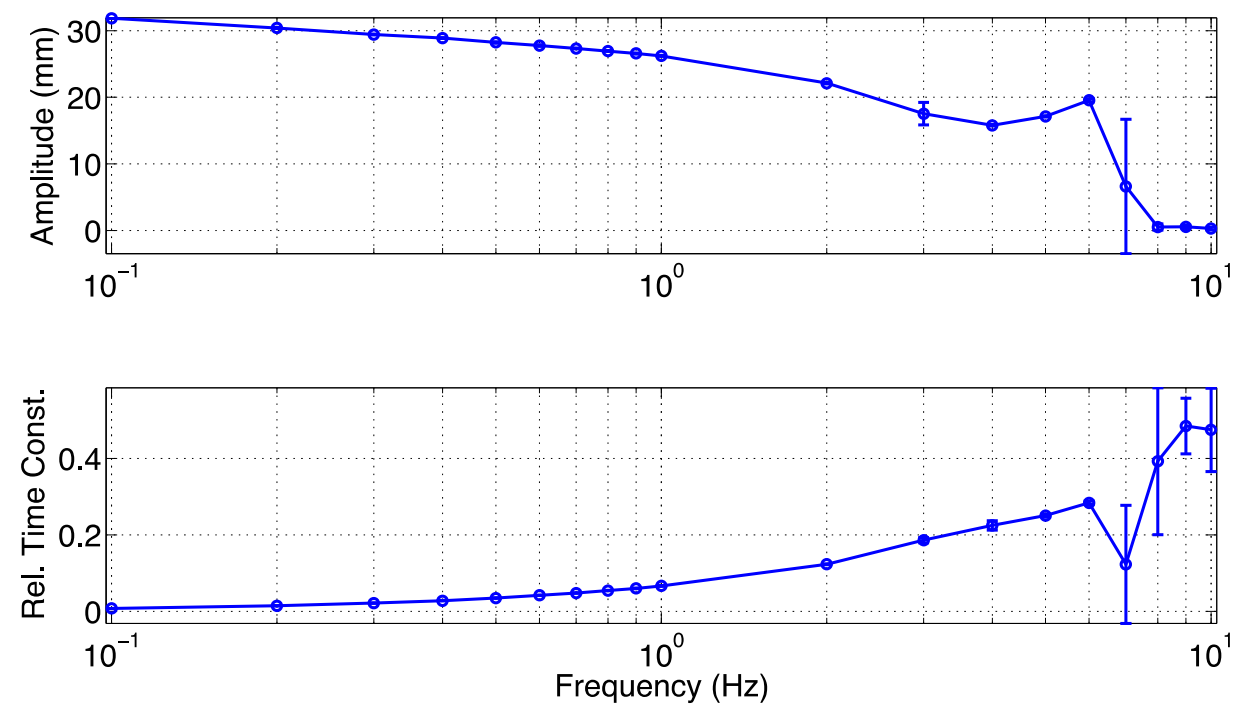

Figure 11. Experimental SWRF plots for the MFC actuation mechanism

The MFC-actuated Flexure Box has a good response for lower frequencies up to $1 \mathrm{~Hz}$, as illustrated by Figure 12(a). For higher frequencies, both the amplitude and RTC start degrading in a similar way as the SMAbased mechanism but for the MFC actuator it occurs for frequencies one order-of-magnitude larger. As input frequency increases even more, shown in Figure 12(b), the structure reaches resonant mode and after that it also reaches a rate-saturation condition, Figure 12(c).
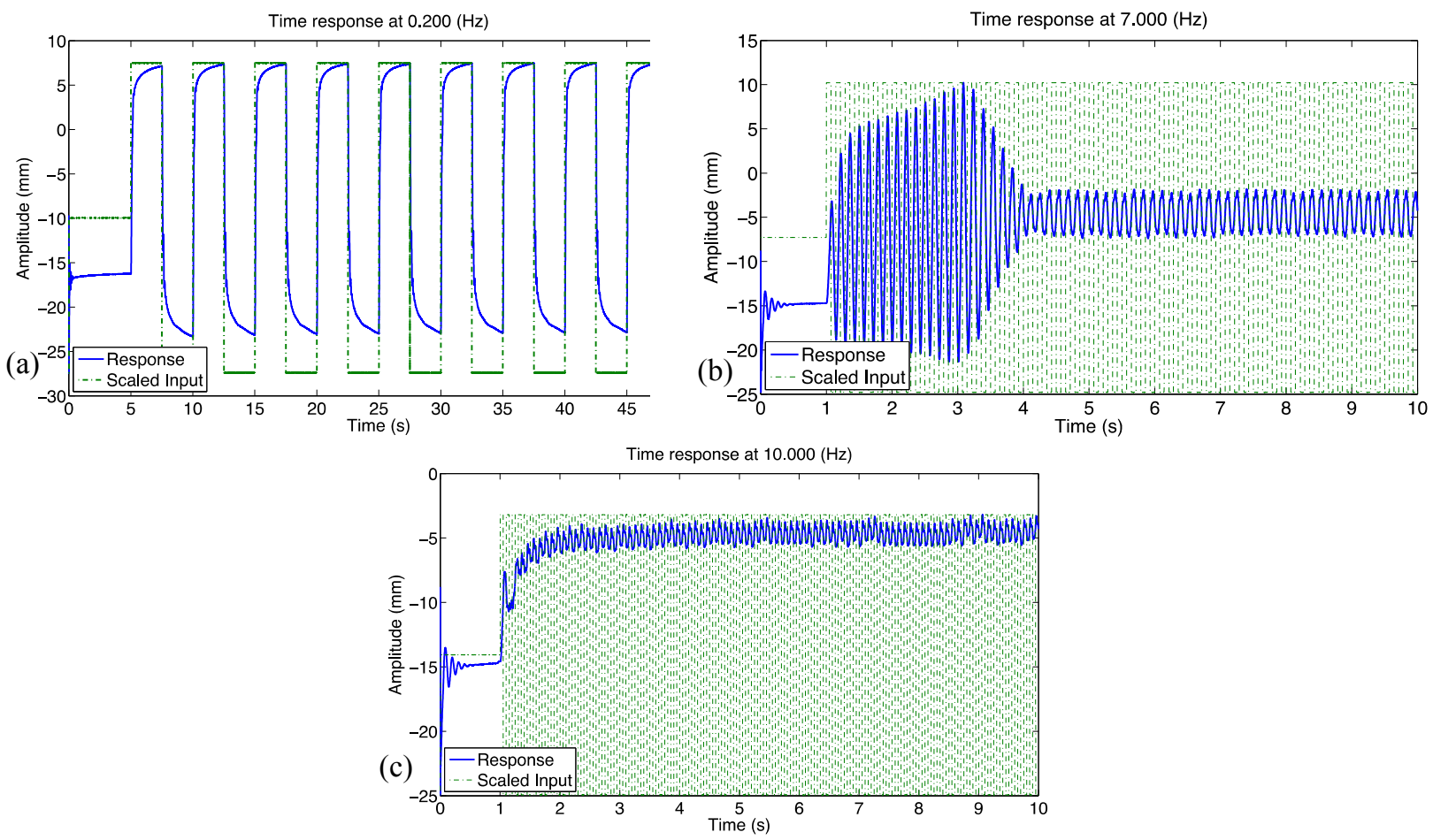

Figure 12. MFC-based actuator time response for (a) $0.2 \mathrm{~Hz}$ (b) $7 \mathrm{~Hz}$ (c) $10 \mathrm{~Hz}$.

By combining the SMA-based and MFC-based mechanisms, the synergistic smart morphing airfoil (SSMA) is formed. The device is tested in a similar way as its individual components and the excitation frequency ranges from $0.05 \mathrm{~Hz}$ to $5 \mathrm{~Hz}$. Figure 13 also has the SWRF for the individual constituents actuators so that a direct comparison can be made across the active devices. 

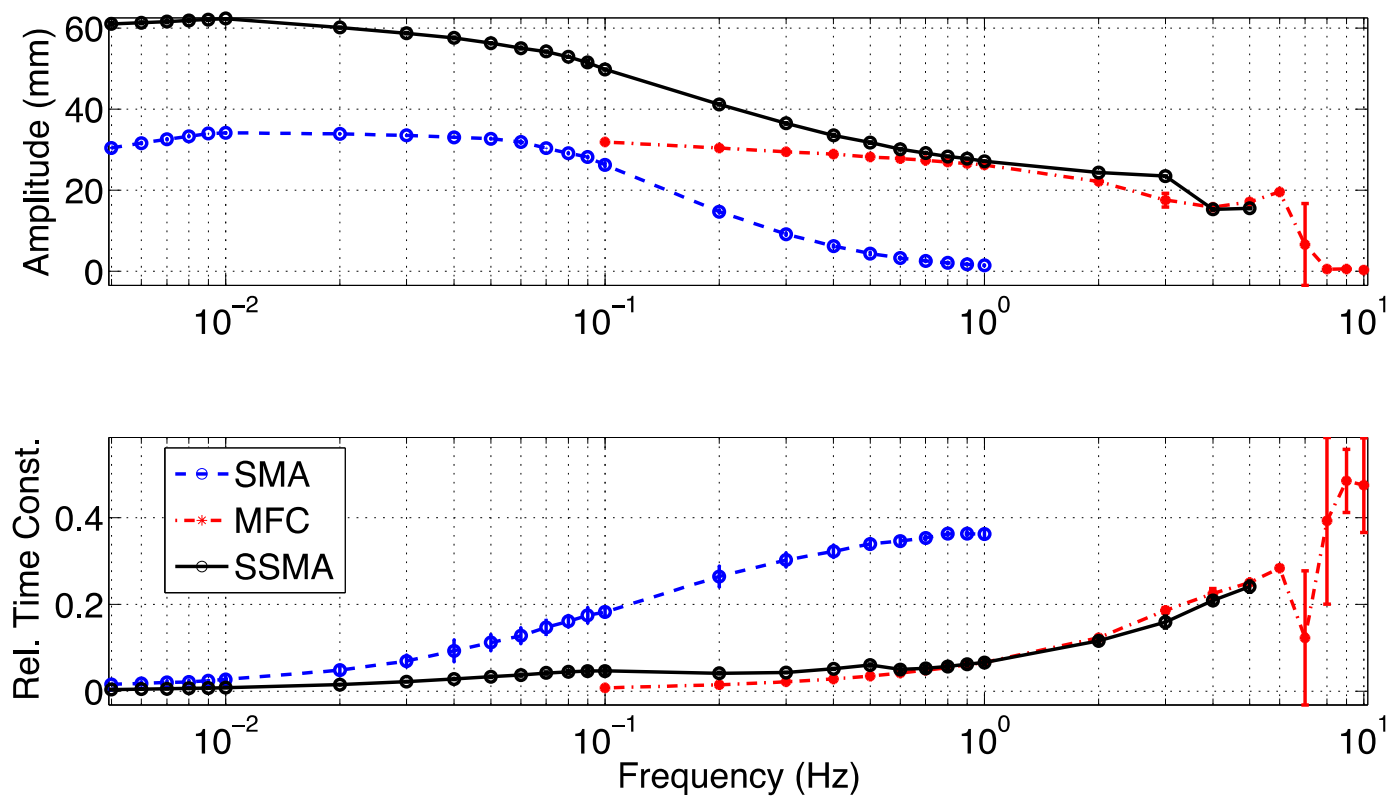

Figure 13. Experimental SWRF plots for the combined actuation mechanism (SMA and MFC)

For low frequency actuation the SSMA system has a good response (high amplitude and low RTC). The amplitude of the SSMA device (solid line) is an addition of the amplitude of each individual active component (dashed lines). It is interesting to note that when the range performance of the SMA decays, the SSMA performance follows the same trend until it matches the MFC-based device (around $0.6 \mathrm{~Hz}$ ). The matching of SWRF plots of the SSMA and MFC proves that only this latter component is effectively responding to the system inputs for frequencies higher than $0.6 \mathrm{~Hz}$. Addtionally, the SSMA relative time constant is not affected by the performance decrease of the SMA actuator (from $0.1 \mathrm{~Hz}$ to $1 \mathrm{~Hz}$ ) this result indicates that the MFC-based mechanism can compensate for decrease in performance of the SMA-based actuator at these frequencies.

\section{Conclusion}

Based on the devices proposed in literature, this paper addresses the need to improve smart structures performance, not by improving material properties or decreasing design complexity of existing concepts, but by combining different smart materials in a synergistic way into a morphing airfoil application. The Synergistic Smart Morphing Aileron (SSMA) is capable of performing a wide range of shape changes in different time scales, surpassing the capabilities of its constituent materials (MFCs and SMAs) as experimentally demonstrated. Not only the range (amplitude of motion) was increased but also the relative time constant (a measure of actuator response speed) was improved for the overall system over the capabilities of the individual concepts.

In addition to range and actuation speed, other considerations from the different concepts were analyzed. The total actuator volume and mass for the SMAs were significantly less than that of the piezo-composite MFCs, efficiently utilizing the SMAs comparatively higher specific work. Although the MFCs showed superior bandwidth, the voltage-controlled nature of the actuators required constant power consumption to maintain the high voltage power supply and thus the position of the actuator. By contrast, the SMAs utilize a phase change to achieve the majority of actuation. So, the position of the SMA actuators remain relatively constant even when the driving circuit is completely powered-off, a fact that is not captured by the SWRF technique but is still significant to powerconscious applications.

The generality of the proposed concept also allows for its extended application as a modular device as part of a larger morphing surface. For example the added degree of freedom in the morphing control surface increases the capability to create more complex shapes over the chord of the airfoil, compared to conventional ailerons. Additionally, the mechanism could be extended for use in a three dimensional wing allowing for variable morphing in the span-wise direction. In both extended applications of the proposed technology it is important to characterize 
how the shape changes affect the flight dynamics. These extensions would presumably allow the vehicle to dramatically increase its flight control authority.

Further studies are needed to evaluate the both advantages and disadvantages of this combination, especially how to develop an adequate controller to simultaneously drive both systems, with power considerations. Also, comparisons with standard technology are necessary to ensure that the current concept does not penalize performance for specific applications of interest.

\section{Acknowledgments}

The authors would like to thank the University of Michgian for the fellowship funding that made this research possible. The authors acknowledge the CNPq Brazilian Research Agency and FAPEMIG through INCT-EIE.

\section{References}

Valasek, J., Morphing Aerospace Vehicles and Structures, Wiley, 2012.

2 Monner, H. P., Hanselka, H., and Breitbach, E., "Development and design of flexible fowler flaps for an adaptive wing," Proceedings of SPIE - The International Society for Optical Engineering, vol. 3326, 1998, pp. 60-70.

3 Sanders, B., Cowan, D., and Scherer, L., "Aerodynamic performance of the smart wing control effectors," Journal of intelligent material systems and structures, vol. 15, 2004, p. 293.

4 Roh, J.-H., Kim, K.-S., and Lee, I., "Shape adaptive airfoil actuated by a shape memory alloy and its aerodynamic characteristics," Mechanics of Advanced Materials and Structures, vol. 16, 2009, pp. 260-274.

5 Anderson, J. D., Fundamentals of Aerodynamics, McGraw-Hill, 2010.

6 Galantai, V., Sofla, A., Meguid, S., Tan, K., and Yeo, W., "Bio-inspired wing morphing for unmanned aerial vehicles using intelligent materials," International Journal of Mechanics and Materials in Design, vol. 8, 2012, pp. 71-79.

7 Kota, S., Osborn, R., Ervin, G., Maric, D., Flick, P., and Paul, D., "Mission adaptive compliant wing-design, fabrication and flight test," Morphing Vehicles, number RTO-MP-AVT-168-18 in. NATO Research and Technology Organization, 2009.

8 Jarali, C. S., Raja, S., and Upadhya, A. R., "Constitutive modeling of SMA SMP multifunctional high performance smart adaptive shape memory composite," Smart Materials and Structures, vol. 19, 2010.

9 Lee, J.-K., and Taya, M., "Modeling for piezoelectric-shape memory alloy composites," Archive of Applied Mechanics, vol. 81, 2011, pp. 629-640.

10 Hines, L., Arabagi, V., and Sitti, M., "Shape memory polymer-based flexure stiffness control in a miniature flapping-wing robot," IEEE Transactions on Robotics, vol. 28, 2012, pp. 987-990.

11 Hagood, N. W., and Bent, A. A., "Development of piezoelectric fiber composites for structural actuation," Collection of Technical Papers - AIAA/ASME Structures, Structural Dynamics and Materials Conference, 1993, pp. 3625-3638.

12 Wilkie, W. K., Bryant, R. G., High, J. W., Fox, R. L., Hellbaum, R. F., Jalink Jr., A., Little, B. D., and Mirick, P. H., "Lowcost piezocomposite actuator for structural control applications," Proceedings of SPIE - The International Society for Optical Engineering, vol. 3991, 2000, pp. 323-334.

13 Barbarino, S., Bilgen, O., Ajaj, R. M., Friswell, M. I., and Inman, D. J., “A Review of Morphing Aircraft,” Journal of Intelligent Material Systems and Structures, vol. 22, 2011, pp. 823-877.

14 Bilgen, O., "Aerodynamic and Electromechanical Design, Modeling and Implementation of Piezocomposite Airfoils," PhD Dissertation, Virginia Tech, 2010.

15 Pankonien, A., and Inman, D. J., "Experimental testing of spanwise morphing trailing edge concept," Proceedings of SPIE The International Society for Optical Engineering, 2013, submitted.

16 Machado, L. G., and Savi, M. A., "Medical applications of shape memory alloys," Brazilian Journal of Medical and Biological Research, vol. 36, 2003, pp. 683-691.

$17 \mathrm{Wu}, \mathrm{M}$. H., and Schetky, L., "Industrial applications for shape memory alloys," Proceedings of the International Conference on Shape Memory and Superelastic Technologies, 2000, pp. 171-182.

18 Andrianesis, K., and Tzes, A., "Design of an anthropomorphic prosthetic hand driven by shape memory alloy actuators," Proceedings of the 2nd Biennial IEEE/RAS-EMBS International Conference on Biomedical Robotics and Biomechatronics, BioRob 2008, 2008, pp. 517-522.

19 Brinson, L. C., "One-dimensional constitutive behavior of shape memory alloys: Thermomechanical derivation with nonconstant material functions and redefined martensite internal variable," Journal of Intelligent Material Systems and Structures, vol. 4, 1993, pp. 229-242.

20 Kim, J.-T., Kim, C., and Lee, S.-R., "Smart composite shell structures with shape memory alloy wires and thin foils," Proceedings of SPIE - The International Society for Optical Engineering, vol. 5649, 2005, pp. 684-691.

21 Barbarino, S., Ameduri, S., Lecce, L., and Concilio, A., "Wing shape control through an SMA-based device," Journal of Intelligent Material Systems and Structures, vol. 20, 2009, pp. 283-296.

22 Kang, W.-R., Kim, E.-H., Jeong, M.-S., Lee, I., and Ahn, S.-M., "Morphing wing mechanism using an SMA wire actuator," International Journal of Aeronautical and Space Sciences, vol. 13, 2012, pp. 58 - 63.

23 Barbarino, S., Pecora, R., Lecce, L., Concilio, A., Ameduri, S., and Calvi, E., "A novel SMA-based concept for airfoil structural morphing," Journal of Materials Engineering and Performance, vol. 18, 2009, pp. 696-705. 
24 Elzey, D. M., Sofia, A. Y. N., and Wadley, H. N. G., "A shape memory-based multifunctional structural actuator panel," International Journal of Solids and Structures, vol. 42, 2005, pp. 1943-1955.

25 Faria, C. T., De Marqui Jr., C., Inman, D. J., and Lopes Jr., V., "Nonlinear dynamic model and simulation of morphing wing profile actuated by shape memory alloys," Conference Proceedings of the Society for Experimental Mechanics Series, vol. 3 , 2012, pp. 21-28.

26 Hambley, A. R., Electrical engineering: principles and applications, Pearson Prentice Hall, 2008. 